\title{
Improved measurement surface for MEG using magnetic-dipole sources and a spherical-multipole expansion
}

\author{
F. Argin, H. Ahrens, and L. Klinkenbusch \\ Institut für Elektrotechnik und Informationstechnik, Christian-Albrechts-Universität zu Kiel, Germany \\ Correspondence to: F. Argin (far@tf.uni-kiel.de)
}

\begin{abstract}
The multipole representation of Magnetoencephalography (MEG) signals is known as a useful tool for distinguishing between magnetic fields arising from the brain and external disturbances. In this contribution we extend this concept and show that a closed double-layer surface with magnetometer probes is better suited to determine the corresponding multipole amplitudes $\alpha_{\mathrm{lm}}$ than a conventional single-layer surface with gradiometers and magnetometer probes. For two different source configurations we show that the $\alpha_{\mathrm{lm}}$ rapidly converge to the exact values. This proof of concept motivates to further optimize the geometry of the double-layer surface and the sensors' positions.
\end{abstract}

\section{Introduction}

Neuronal activity of the human brain involves electrical currents which give rise to a magnetic field outside of the brain. Since the typical intensity of the field is in the femtotesla range its measurement requires a lot of effort. Current MEG techniques involve magnetically shielded environments and Superconducting Quantum Interference Devices (SQUIDs). From the measured magnetic field the field sources can be estimated by different algorithms. Obviously the reduction of noise and the elimination of external disturbances improve the estimation of the field sources. Assuming divergencefree current sources only, the magnetic field outside the brain can be described by a scalar magnetic potential which will be represented by a spherical-multipole expansion. It has been shown that this technique allows to separate external disturbances from internal sources (Signal Space Separation) (Taulu et al., 2004). We extend this idea and introduce a closed double-layered measurement surface. The field within this source-free enclosure can be uniquely determined if Neumann or Dirichlet boundary data are given on its surface. From the knowledge of its boundary values the magnetic field is uniquely determined also outside of the en- closure, particularly also in the magnetically homogeneous brain outside of the sources. The field measured on this new measurement surface is represented by a spherical-multipole expansion which conveniently can be used to interpret the biomagnetic field as well as to optimize the shape of the measurement surface and the sensors' positions.

In Sect. 2 we demonstrate that the neuronal activity, that is, the divergence-free currents which produce the biomagnetic field can be described by magnetic dipoles which leads us to a most simple characterization of the forward problem. Since the domain outside the brain is free of sources, we deduce a spherical-multipole expansion for that curl-free magnetic field arising from sources in the brain. In Sect. 3 we show how the measurement surface has been designed to ensure that we are able to determine the multipole-amplitudes uniquely. Section 4 illustrates how the single-layer and double-layer surfaces were implemented, as well as the setup of the system of linear equations for numerically determining the spherical-multipole amplitudes $\alpha_{\mathrm{lm}}$.

Numerical results for a single-layer measurement surface with symmetric axial first order gradiometer as well as magnetometer data, and for a closed double-layer surface using magnetometer data only are finally presented in Sect. 5 .

\section{Forward problem}

\subsection{Magnetic dipole moments}

While the brain is processing information, small electric currents flow inside the neural system producing a magnetic field outside of the head. This extremely weak magnetic field can be recorded by suitable MEG devices (Hämäläinen et al., 1993). Because of the complexity of the neuronal system, the exact current flows are difficult to describe. Without going into detail of the brain physiology, we shall point out a few of the relevant aspects leading to MEG signals. It is 


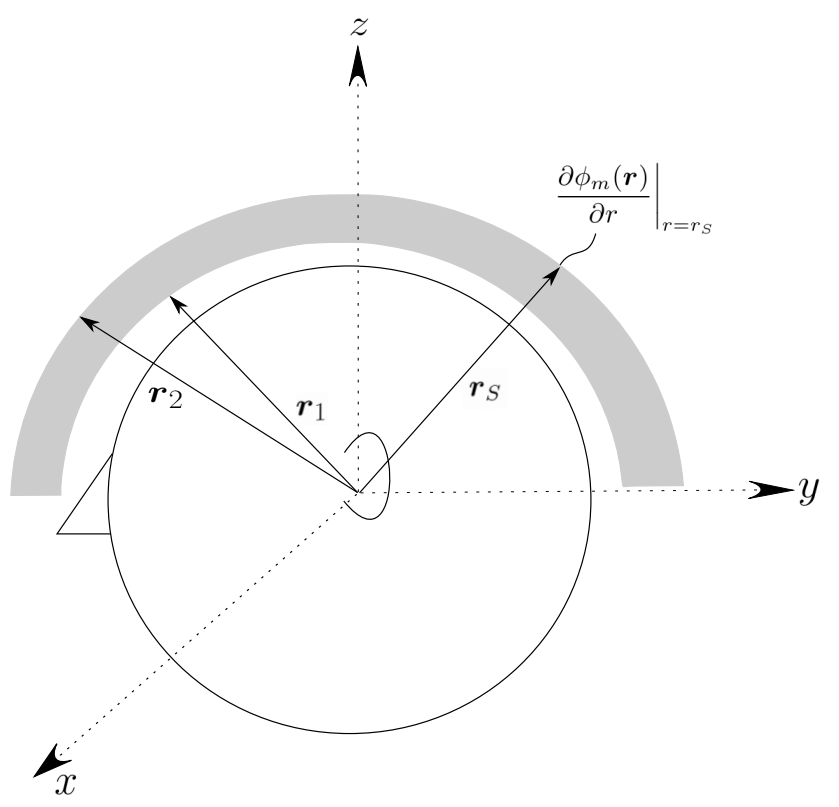

Fig. 1. Schematic view of a simplified head model and a closed double-layer surface. The radius of the first layer is $\boldsymbol{r}_{1}$ and the radius of the second one is $\boldsymbol{r}_{2}$. The measurement surface is the entire (closed) surface of the gray domain. The vector $\boldsymbol{r}_{s}$ points on that surface where $\left.B_{r} \sim \frac{\partial \phi_{m}(\boldsymbol{r})}{\partial r}\right|_{r=r_{S}}$ is measured.

assumed that the main source of MEG signals are excitatory postsynaptic potentials (EPSPs) (Nunez, 1981) in the input part of the neurons. The EPSPs cause current flows inside the excited neurons (Gloor, 1985), which - due to the commonly presumed conservation of charge at each time - must be balanced by an electrical counterflow outside of the neuron (Baillet et al., 2001). Usually, the intracellular current density $\boldsymbol{J}_{p}(\boldsymbol{r})$ is called primary current density while the extracellular part $\boldsymbol{J}_{V}(\boldsymbol{r})$ is referred to as the volume current density.

The total electric-current density $\boldsymbol{J}(\boldsymbol{r})$ is

$\boldsymbol{J}(\boldsymbol{r})=\boldsymbol{J}_{p}(\boldsymbol{r})+\boldsymbol{J}_{V}(\boldsymbol{r})$.

It is commonly presumed that at any time there is no accumulation of charge in the neuronal system, hence the total current must be divergence-free according to

$$
\nabla \cdot J(r)=0
$$

which we may electrically interpret as a closed current circuit. Hence it is possible to represent $\boldsymbol{J}(\boldsymbol{r})$ by a suitable number of located magnetic dipoles described by corresponding dipole moments $\boldsymbol{m}$. For a given current density located in $\Delta V$ it holds that

$\left.\boldsymbol{m}\right|_{\Delta V}=\frac{\mu_{0}}{2} \int_{\Delta V} \boldsymbol{r} \times \boldsymbol{J}(\boldsymbol{r}) \mathrm{d}^{3} r$ which in case of a single closed current circuit with a (directed) area $\boldsymbol{F}$ and a current strength $I$ has the solution

$\boldsymbol{m}=\mu_{0} \boldsymbol{F} I$.

Commonly tens of thousands of neurons have to be activated at the same time to have measurable MEG signals, therefore the magnetic dipole moment can be understood as an equivalent source of the net effect of current circuits covering several cubic centimeters of activated brain tissue.

In general, the frequencies for brain signals are in the range of or below $100 \mathrm{~Hz}$, hence we can neglect the time derivatives $\sigma|\partial \boldsymbol{E} / \partial t|$ and $|\partial \boldsymbol{B} / \partial t|$ in Maxwell's equations (Hämäläinen et al., 1993). This leads us to the following quasi-static approximation of Maxwell's equations for the magnetic field produced by a magnetic dipole moment $\boldsymbol{m}$ located at $\boldsymbol{r}_{0}$

$\boldsymbol{B}(\boldsymbol{r})=\frac{\mu_{0}}{4 \pi} \frac{3\left(\boldsymbol{r}-\boldsymbol{r}_{0}\right)\left(\boldsymbol{m} \cdot\left(\boldsymbol{r}-\boldsymbol{r}_{0}\right)\right)}{\left|\boldsymbol{r}-\boldsymbol{r}_{0}\right|^{5}}-\frac{\boldsymbol{m}}{\left|\boldsymbol{r}-\boldsymbol{r}_{0}\right|^{3}}$.

\subsection{Multipole expansion}

Assuming that the domain outside of the head is homogeneous and free of sources, Ampère's law in the magnetoquasi-static approximation reads

$\nabla \times B(r)=0$

Outside of the head the magnetic field produced by closedcurrent circuits (or by magnetic dipoles) in the head consists of curl-free fields only, hence it is always possible to deduce that magnetic field from a scalar magnetic potential $\phi_{m}(\boldsymbol{r})$ according to

$\boldsymbol{B}(\boldsymbol{r})=-\mu_{0} \nabla \phi_{m}(\boldsymbol{r})$.

With $\boldsymbol{\nabla} \cdot \boldsymbol{B}=0$ we easily derive the Laplace equation

$\Delta \phi_{m}(\boldsymbol{r})=0$

In spherical coordinates $(r, \vartheta, \varphi)$ the general solution can be found as a spherical-multipole expansion

$\phi_{m}(\boldsymbol{r})=\sum_{l=0}^{\infty} \sum_{m=-l}^{l}\left[\alpha_{\operatorname{lm}} \frac{Y_{\operatorname{lm}}(\theta, \phi)}{r^{l+1}}+\beta_{\operatorname{lm}} r^{l} Y_{\operatorname{lm}}(\theta, \phi)\right]$

with multipole-amplitudes (expansion coefficients) $\alpha_{1 \mathrm{~m}}$ and $\beta_{1 \mathrm{~m}}$. The surface-spherical harmonics are related to associated Legendre functions and harmonic functions by

$Y_{\operatorname{lm}}(\theta, \phi)=\sqrt{\frac{2 l+1}{4 \pi} \frac{(l+1) !}{(l-1) !}} P_{l}^{m}(\cos \theta) e^{j m \phi}$.

As can be seen from Eq. (9) only the part in the multipole expansion belonging to $\alpha_{1 \mathrm{~m}}$ remains regular for $r \rightarrow \infty$. Consequently, that part can be interpreted as the magnetic field arising from magnetic sources inside the head. On the other hand, only the part in the multipole expansion belonging to 


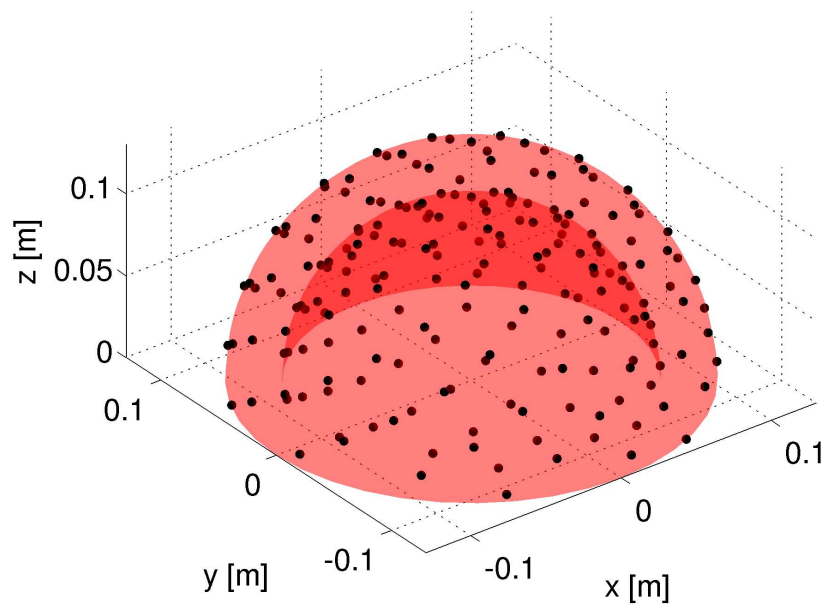

Fig. 2. Closed double-layer surface with isotropically distributed pointlike magnetometers (black dots). The radius of the first layer is $r_{1}=10 \mathrm{~cm}$ and the radius of the second layer is $r_{2}=13 \mathrm{~cm}$.

$\beta_{\operatorname{lm}}$ remains finite for $r \rightarrow 0$, therefore this part represents the magnetic field arising from sources outside the head.

In this paper we consider sources inside the head only and neglect external (interfering) sources, hence only the field part with the $\alpha_{\mathrm{lm}}$ is relevant.

\section{Implementation of the measurement surfaces}

The scalar potential $\phi(r)$ within the closed measurement surface (gray domain in Fig. 1) can be determined uniquely, if the corresponding Neumann $\left(\partial \phi(\boldsymbol{r}) /\left.\partial n\right|_{\boldsymbol{r}=\boldsymbol{r}_{S}}\right)$ or Dirichlet $\left(\left.\phi(r)\right|_{r=r_{S}}\right)$ boundary data are known. In this paper, the measurement surface consists of two connected hemispheres with different radii (Fig. 2). From Eq. (7) we obtain

$\left.B_{r}(\boldsymbol{r})\right|_{\boldsymbol{r}=\boldsymbol{r}_{S}}=-\left.\mu_{0} \frac{\partial \phi_{m}(\boldsymbol{r})}{\partial r}\right|_{\boldsymbol{r}=\boldsymbol{r}_{S}}$,

on the hemispheres, that is, the solution of the Neumann problem is equivalent to finding the normal component of the magnetic field of the measurement surface. Practically, we measure the radial component at isotropically distributed points on a closed double-layer surface (Fig. 2). The results will be compared to those ones obtained from a single measurement surface (Fig. 3) on which we assume given Cauchy data, i.e., $\left.\boldsymbol{B}_{r}(\boldsymbol{r})\right|_{\boldsymbol{r}=\boldsymbol{r}_{S}}$ and simultaneously $\left.\left(\partial \boldsymbol{B}_{r}(\boldsymbol{r}) / \partial r\right)\right|_{\boldsymbol{r}=\boldsymbol{r}_{S}}$. The latter configuration is the standard procedure in MEG measurement, where magnetometers as well as gradiometers are taken into account.

To compare the results obtained from the different measurement surfaces, we solve the forward problem analytically from Eq. (5) for two simple source configurations. For the single-layer configuration the positions of the magnetometers and gradiometers are alternating. The radius of the single-layer surface as well as of the first layer of the

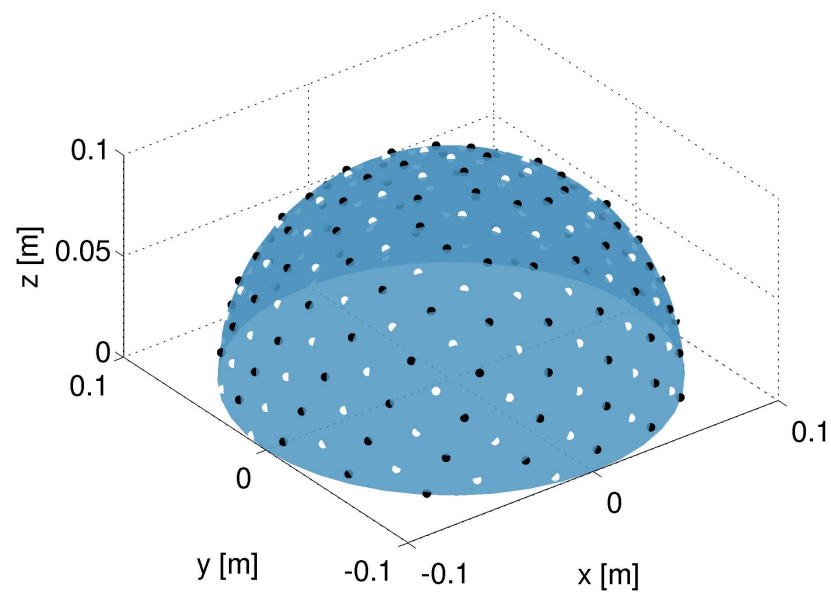

Fig. 3. Single-layer surface with isotropically distributed pointlike magnetometers (black dots) and pointlike gradiometers (white dots). The units of the axes are in meters.

closed surface is $r_{1}=10 \mathrm{~cm}$. For the second layer of the closed double-layer surface the radius has been chosen to $r_{2}=13 \mathrm{~cm}$.

Next, the magnetic field $\boldsymbol{B}(\boldsymbol{r})$ of the mentioned simple source configuration is expressed as a spherical-multipole expansion Eq. (9) with multipole amplitudes $\alpha_{\operatorname{lm}}$ only. For each of the field values at the probes' positions we set up a corresponding spherical-multipole expansion. Doing this for each probe, we end up with a system of linear equations. Its solution yields the multipole amplitudes of the reconstructed magnetic field, which then is compared to the original (true) field value at the probes' positions.

The first of the source configurations consists of a magnetic dipole located at the origin of the coordinate system and polarized in the $z$-direction, while for the second one we place the magnetic dipole at $\boldsymbol{r}=5 \mathrm{~cm} \hat{z}$ polarized in the $x$ direction. Arranging the multipole expansions for each of the measurement points in rows, the system of linear equations reads as

$$
\underbrace{\left(\begin{array}{cccc}
\Omega_{1,-1}^{1} & \Omega_{1,0}^{1} & \ldots & \Omega_{l, l}^{1} \\
\Omega_{1,-1}^{2} & \Omega_{1,0}^{2} & \ldots & \Omega_{l, l}^{2} \\
\vdots & \ddots & \ddots & \vdots \\
\Omega_{1,-1}^{N} & \Omega_{1,0}^{N} & \ldots & \Omega_{l, l}^{N}
\end{array}\right)}_{=\mathbf{A}} \underbrace{\left(\begin{array}{c}
\alpha_{1,-1} \\
\alpha_{1,0} \\
\vdots \\
\alpha_{l, l}
\end{array}\right)}_{=\boldsymbol{\alpha}}=\underbrace{\left(\begin{array}{c}
B_{r}\left(\boldsymbol{r}_{1}\right) \\
B_{r}\left(\boldsymbol{r}_{2}\right) \\
\vdots \\
B_{r}\left(\boldsymbol{r}_{N}\right)
\end{array}\right)}_{=\boldsymbol{b}} .
$$

Here, $\Omega_{l, m}^{k}=\frac{(l+1) Y_{\operatorname{lm}}\left(\theta_{k}, \phi_{k}\right)}{r_{k}^{l+2}}, \alpha_{1 \mathrm{~m}}$ are the spherical-multipole amplitudes while $B_{r}\left(\boldsymbol{r}_{k}\right)$ denotes the radial component of the measured magnetic field at the $k$-th measurement point.

The exact solution of the system of linear equations might be obtained only if it is at least squared and of full rank. Because the number of columns of $\mathbf{A}$ are depending on the multipole order $l$, the number of necessary rows (measurement points) is given by $N=(l+1)^{2}-1$. 


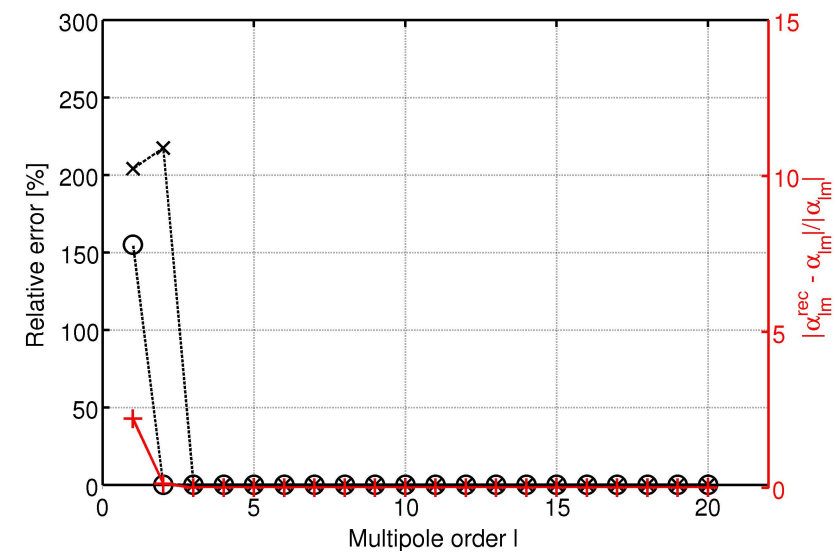

Fig. 4. Relative reconstruction error of the components $B_{r}$ (black o) and $B_{\theta}($ black $\times)$ on a closed double-layer surface and of the original multipole expansion (red) as a function of the order $l$ of the multipole expansion. Source: Dipole at the origin, polarized in $z$-direction.

For the double-layer surface we have to combine the data of the inner and the outer layer. The single-layer surface consists of magnetometers and (symmetrically arranged) gradiometers. A gradiometer provides a first order derivation of $\boldsymbol{B}(\boldsymbol{r})$ in radial direction at the measurement point, thus the elements of the coefficient matrix of the gradiometer probe are

$\tilde{\Omega}_{l, m}^{k}=\frac{\frac{(l+1) Y_{\operatorname{lm}}\left(\theta_{k}, \phi_{k}\right)}{r_{g_{2}, k}^{l+2}}-\frac{(l+1) Y_{\operatorname{lm}}\left(\theta_{k}, \phi_{k}\right)}{r_{g_{1}, k}^{l+2}}}{r_{g_{2}, k}-r_{g_{1}, k}}=\frac{\Omega_{l, m}^{g_{2}, k}-\Omega_{l, m}^{g_{1}, k}}{\Delta r_{g, k}}$

while the elements of the right hand side read as

$\tilde{B}_{r}\left(\boldsymbol{r}_{k}\right)=\frac{B_{r}\left(\boldsymbol{r}_{g_{2}, k}\right)-B_{r}\left(\boldsymbol{r}_{g_{1}, k}\right)}{\Delta r_{g, k}}$.

Here, $r_{g_{1}, k}$ denotes the distance of the first coil and $r_{g_{2}, k}$ of the second one of the axial gradiometer. Thus the combination of magnetometer and gradiometer probes leads us to the following system of linear equations

$$
\left(\begin{array}{cccc}
\tilde{\Omega}_{1,-1}^{1} & \tilde{\Omega}_{1,0}^{1} & \ldots & \tilde{\Omega}_{l, l}^{1} \\
\Omega_{1,-1}^{2} & \Omega_{1,0}^{2} & \ldots & \Omega_{l, l}^{2} \\
\vdots & \ddots & \ddots & \vdots \\
\tilde{\Omega}_{1,1}^{N-1} & \tilde{\Omega}_{1,0}^{N-1} & \ldots & \tilde{\Omega}_{l, l}^{N-1} \\
\Omega_{1,-1}^{N} & \Omega_{1,0}^{N} & \ldots & \Omega_{l, l}^{N}
\end{array}\right)\left(\begin{array}{c}
\alpha_{1,-1} \\
\alpha_{1,0} \\
\vdots \\
\alpha_{l, l}
\end{array}\right)=\left(\begin{array}{c}
\tilde{B}_{r}\left(\boldsymbol{r}_{1}\right) \\
B_{r}\left(\boldsymbol{r}_{2}\right) \\
\vdots \\
\tilde{B}_{r}\left(\boldsymbol{r}_{N-1}\right) \\
B_{r}\left(\boldsymbol{r}_{N}\right)
\end{array}\right)
$$

For estimating the quality of the reconstructed fields we define a relative error according to

$\operatorname{err}_{\psi}=\frac{\sum_{k=1}^{N}\left|B_{\psi}^{k}-B_{\psi}^{k(r e c)}\right|}{\sum_{k=1}^{N}\left|B_{\psi}^{k}\right|}, \psi \in\{\mathrm{r}, \theta, \phi\}$

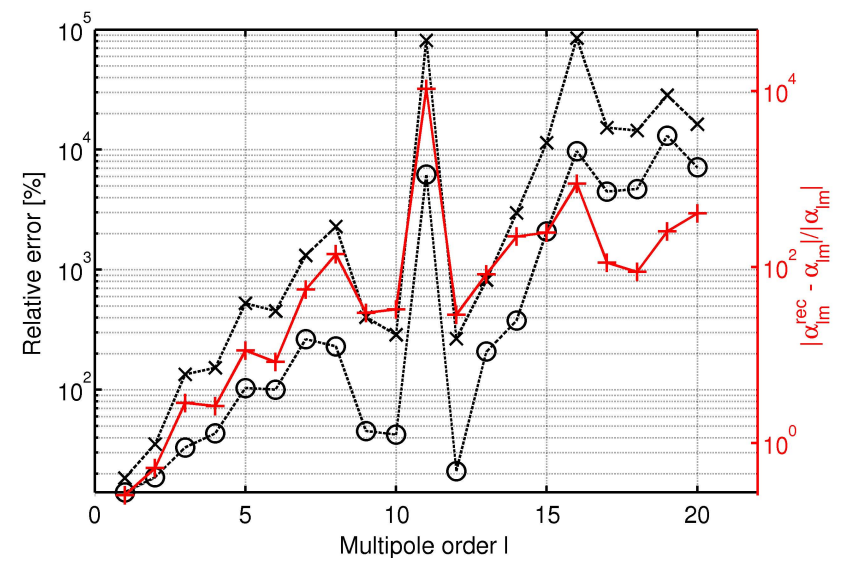

Fig. 5. Relative reconstruction error of the components $B_{r}$ (black o) and $B_{\theta}($ black $\times)$ on a single-layer surface and of the original multipole expansion (red) as a function of the order $l$ of the multipole expansion. Source: Dipole at the origin, polarized in $z$-direction.

\section{Numerical results}

The first example concerns the magnetic field produced by a magnetic dipole moment $\boldsymbol{m}=m \hat{z}$ located at the origin of the coordinate system. For this configuration $B_{\phi}$ vanishes, and we are investigating the relative errors of the $B_{r}$ and $B_{\theta}$ components only. Moreover, in this case the only non-zero multipole amplitude is $\alpha_{1,0}$. In the following text we will denote the analytically obtained (exact) multipole amplitudes as $\alpha_{\mathrm{lm}}$ while the multipole amplitudes obtained from the reconstruction procedure are referred to as $\alpha_{\mathrm{lm}}^{\mathrm{rec}}$.

The black curves in Fig. 4 show that the measurement on the closed double-layer surface yields an excellent reconstruction of the magnetic field at the measurement points with a few multipole amplitudes considered. The magneticfield error which can be estimated by the summed difference between corresponding multipole amplitudes of the reconstructed field and the original field, also shows an excellent and stable behavior when a few multipole amplitudes are considered. In contrast, for the single-layer measurement surface the deviation between reconstructed and original fields even diverge with an increasing order of the multipole expansion as we can deduce from the results shown in Fig. 5.

The second example was performed with a magnetic dipole moment $\boldsymbol{m}=m \hat{x}$ located at $x=0, y=0, z=5 \mathrm{~cm}$. Since the number of relevant multipole amplitudes increases if the sources are located off the origin, also the numbers of multipole amplitudes needed for the reconstruction increase. However, we again observe from Fig. 6 that the magnetic field reconstructed from the measurement on the closed double-layer surface constantly converges to the original field.

Note that reconstruction of the tangential components $B_{\theta}(\boldsymbol{r})$ and $B_{\phi}(\boldsymbol{r})$ also is converging, however, not as fast 


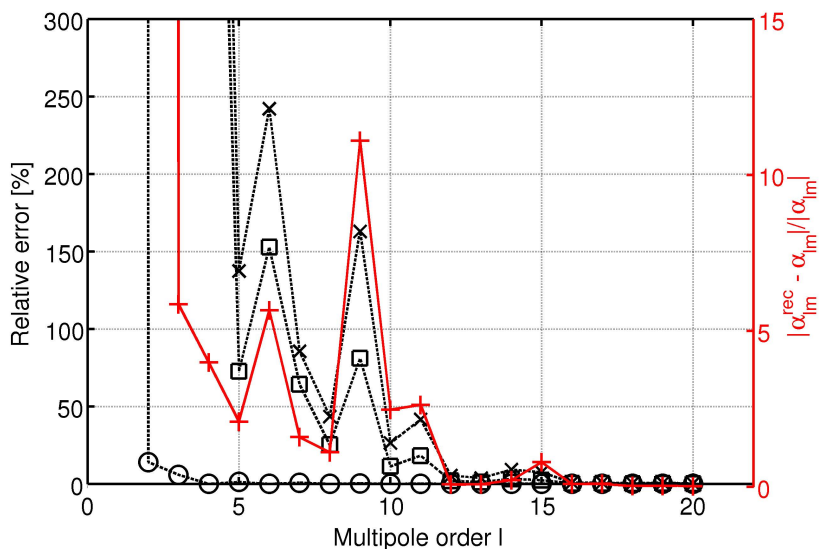

Fig. 6. Relative reconstruction error of the components $B_{r}$ (black $\circ), B_{\theta}($ black $\times)$, and $B_{\phi}$ (black $\square$ ) on a closed double-layer surface and of the original multipole expansion (red) as a function of the order $l$ of the multipole expansion. Source: Dipole at $\boldsymbol{r}=5 \mathrm{~cm}$ $\hat{z}$, polarized in $x$-direction.

as for the radial component. The reason for this behavior can simply be found in the underlying numerical procedure, as the corresponding system of linear equations has been solved with respect to the radial component at the measurement points. Again, the reconstruction of the magnetic field from measurements on a single-layer measurement surface fails (see Fig. 7), i.e., it diverges with an increasing number of multipole amplitudes considered.

\section{Conclusions}

It has been shown that the use of a closed double-layer measurement surface is excellently suited to reconstruct the spherical-multipole expansion of a divergence-free quasistatic magnetic field. In contrast, the same method equivalently applied to the conventionally used single-layer surface with magnetometers and gradiometers showed no convergence. The method can be applied to Magnetoencephalography as well as to Magnetocardiography. Particularly, it is best suited for the currently developed new type of magnetic field sensors based on magnetoelectric composite materials.

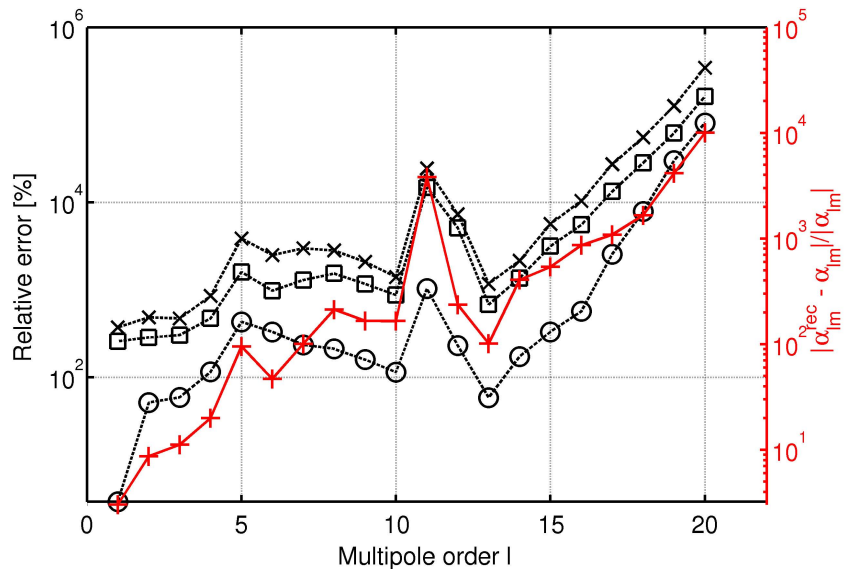

Fig. 7. Relative reconstruction error of the components $B_{r}$ (black $\circ), B_{\theta}($ black $\times)$, and $B_{\phi}$ (black $\square$ ) on a single-layer surface and of the original multipole expansion (red) as a function of the order $l$ of the multipole expansion. Source: Dipole at $\boldsymbol{r}=5 \mathrm{~cm} \hat{z}$, polarized in $x$-direction.

Acknowledgements. The work was supported by the Deutsche Forschungsgemeinschaft within the SFB855: Magnetoelectric composite materials - Future biomagnetic interface.

\section{References}

Baillet, S., Mosher, J. C., and Leahy, R. M: Electromagnetic brain mapping, IEEE Signal. Proc. Mag., 18, 14-30, 2001.

Gloor, P.: Neuronal generators and the problem of localization in electro-encephalography: Application of volume conductor theory to electroencephalography, J. Clin. Neurophysiol., 2, 327 354, 1985.

Hämäläinen, M., Hari, R., Ilmoniemi, R. J., Knuutila, J., and Lounasmaa, O. V.: Magnetoencephalography - Theory, instrumentation, and applications to noninvasive studies of the working human brain, Rev. Mod. Phys., 65, 413-497, 1993

Nunez, P. L.: Electric Fields of the Brain, New York:Oxford, USA, 1981.

Taulu, S. and Matti, K.: Presentation of electromagnetic multichannel data: The signal space separation method, J. Appl. Phys., 97 124905-124905-10, 2005.

Taulu, S., Kajola, M., and Simola, J.: Suppression of Interference and Artifacts by the Signal Space Separation Method, Brain Topogr., 16, 269-275, 2004. 